\title{
CLINICAL REFLECTION \\ Bewildered and alone: supporting people with cognitive impairment on COVID isolation wards
}

\author{
Martha Finnegan (다 \& Elaine Greene
}

\begin{abstract}
Martha Finnegan is a Fellow in
Young Onset Dementia at St James's and Tallaght Hospitals, Dublin and formerly a senior registrar in liaison psychiatry for the elderly. She is based in the Department of Psychiatry for the Elderly at St James's Hospital, Dublin, Ireland. Elaine Greene is a Consultant Psychiatrist in Liaison Psychiatry for the Elderly in the Department of Psychiatry for the Elderly at St James's Hospital, Dublin and Associate Clinical Professor of Psychiatry at Trinity College Dublin, Ireland.

Correspondence Martha Finnegan. Email: mfinneg@tcd.ie
\end{abstract}

First received 27 0ct 2021

Accepted 8 Nov 202

\section{Copyright and usage}

(C) The Author(s), 2021. Published by Cambridge University Press on behalf of the Royal College of Psychiatrists. This is an Open Access article, distributed under the terms of the Creative Commons Attribution licence (https://creativecommons. org/licenses/by/4.0//, which permits unrestricted re-use, distribution, and reproduction in any medium, provided the original work is properly cited.

\section{SUMMARY}

Managing isolation protocols for distressed, cognitively impaired COVID-19-positive patients presented a range of new challenges to our liaison psychiatry for the elderly service. In this article we present some of the scenarios we have experienced, our own reflections on the needs of this specific group and how this has challenged us in terms of tolerating risk, prescribing off-label, collaborating with distressed colleagues, professional boundaries and being creative in non-pharmacological interventions.

\section{KEYWORDS}

COVID-19; liaison; elderly; polypharmacy; pandemic.

In our practice in liaison psychiatry for the elderly in Ireland's largest teaching hospital we continued our service throughout the pandemic, supporting COVID-19 teams to manage the psychiatric needs of older in-patients in COVID triage wards, isolation units and the intensive care unit. This included patients with cognitive impairment or delirium admitted with COVID-19-related illness or who developed the illness during their admission.

During the pre-vaccination period of the COVID19 pandemic (March 2020 to January 2021), the majority of the service's consultations for COVIDpositive patients were conducted remotely. Multiple telephone discussions were held with teams, ward staff, families and other involved clinicians. Video calls with patients were almost uniformly unsuccessful and usually resulted in their increased bewilderment and no progress in history taking. During surges in COVID-19 cases, the service quickly changed to provide the kind of rapid, informal, often out-of-hours consultant-to-consultant advice that is usually anathema to consultation-liaison specialties. The pandemic upended usual systems of thoughtful referral processes, multidisciplinary teamwork and boundaried relationships. Our colleagues in respiratory, infectious, emergency and intensive medicine had suddenly shouldered extraordinary risk and burden. Supporting their tasks became the overriding concern across the hospital. Suddenly it mattered that interns should have your personal mobile number, if they are the ones in full PPE (personal protective equipment) and you are not.

\section{Changing views on risk}

Perhaps counterintuitively, by far the most distressing, most challenging situations involved those COVID patients who were most physically healthy, perhaps even asymptomatic. Many mildly cognitively impaired patients had some sense that they were being prevented from doing what they had been able to do previously and became very distressed. In contrast, for our patients who were either in respiratory distress, fatigued or worse, agitation subsided but distress remained evident. Compliance with oxygen therapy, proning or sustaining intravenous access was sometimes impossible without significantly higher or more frequent doses of sedating psychotropics. Our analysis of risks versus benefits when prescribing was simplified by the sheer necessity of acting to address immediate problems. In some cases polypharmacy, the mortal enemy of the old age psychiatrist, became a necessary evil. There was no greater pressure than usual to prescribe from the COVID teams. This was possibly because, where COVID teams felt additional psychotropics were required, they did not hesitate to prescribe them. Being at the coalface meant they had to become self-sufficient at managing almost any human condition with distant advice.

\section{The depleted arsenal of care}

For all staff, being unable to enter isolation rooms except for clinical activities transformed how we helped the patients in our care. The practical reality of new rules meant that radios could not be turned off on request, television channels changed, clothes helped on or off, or sitting positions changed for longer periods. Waiting for assistance can feel like an eternity, but for a forgetful, disoriented person it may mean feeling hopelessly alone or abandoned. Even when a staff member could enter, the necessary PPE made them look like 
something out of a sci-fi movie. Additionally, the treatment protocols meant that staff had to remain far more aloof and distant than in pre-COVID times. Our colleagues across nursing, healthcare assistance and housekeeping were robbed of their unique healing power - their ability to immediately resolve most minor discomforts and meet simple requests. For many of them, this was deeply upsetting. One household staff member told us she could not believe she could no longer pat the arm of her patients when dropping off their dinners, a small gesture she had performed about 60 times per workday for nearly 30 years. Other colleagues described a feeling of moral anguishin being unable to comfort a patient - rarely out of concerns for one's own health but concern for transmission to other patients, vulnerable family members or colleagues. On some days, wards seemed soaked in distress, reflecting the emotions of patients and staff.

Our non-pharmacological arsenal seemed to have been ransacked - reliable ward-based repetitive activity for patients with more advanced cognitive impairment was extremely limited. Even asking families to drop favourite items from home in the drop-off zones was fraught as items such as textiles could not be sanitised and returned. In one ward a patient with moderate dementia simply could not be safely contained in their isolation room despite escalating doses of psychotropics, with such persistent and extreme expressions of distress at times that healthcare assistants stationed at the isolation room door could tolerate only 15minute shifts. On the worst day, staff cleared the ward corridor during a quiet time of day and dressed the patient in full PPE to allow a therapeutic wander that de-escalated much of the agitation. It is hard, in vaccinated times, to appreciate how significant this decision was for staff and the patient.

\section{Sharing burdens and balancing needs}

While unimpaired isolated patients could, if well enough, keep in touch by phone and text all day with those on the outside, families removed from cognitively impaired patients who were sick, dying or well but distressed could only rely on telephone contact with the ward and teams. One family member of a relatively stable older patient described their overnight rota for a telephone vigil in the family home. The stakes were high on every phone call and junior doctors described the pressure of communicating with great accuracy in situations where they were fatigued and the clinical situation was inherently unpredictable. In this context, we were unsurprised to experience an expectation that where we were involved with a COVID patient's care, we would liaise more extensively with the family than in normal times. There was a sense of relief among medical teams that for at least one or two of the patients on their ward, the burden of family communication was shared.

Balancing the needs of our cognitively impaired COVID patients with the legitimate needs of other patients - ours and others - for care, or avoidance of transmission, made every interaction a deliberate choice. Choosing to go to the COVID ward at a particular time was as weighty as choosing not to go. At times, when patients well-known to our co-workers in community mental health teams were admitted with COVID, balancing the team's and family's desire for them to be seen often, or to pass regular messages of care to them, with a sometimes limited clinical imperative for their ongoing review, needed consideration. Cohorting COVID-positive cognitively impaired patients in a room with nonimpaired (but perhaps physically sicker) COVID patients also needed thought as it seemed to invoke a sense of responsibility in cognitively intact peers to comfort or supervise their ward neighbour, so much so that it seemed unfairly burdensome or even manipulative, when it worked well.

\section{Longer-term repercussions}

As soon as vaccination commenced, although community and hospital restrictions remained unchanged, we felt an expectation (whether from others or ourselves) of increasing service to COVID wards, including more in-person assessments. For some of us, a new challenge of restraint from over-involvement arose, perhaps from relief at being needed, being involved or contributing after a long suspension of normal clinical life and purpose. We joked that old age psychiatrists simply could not be diverted from their perseverative efforts to 'have a little chat'.

The wider repercussions of this time are yet to be felt, for everyone who experienced it. It may be too soon for reflection. Our service was stretched and will remodel to a new shape. For us, remembering that porous boundaries and informal advice have their time and place, and, on testing, did not catastrophically dilute our guiding principles, may be useful to prepare our service for the future.

\section{Author contributions}

Both authors conceived of and collaborated on this Clinical Reflection, first draft by M.F.

\section{Funding}

This work received no specific grant from any funding agency, commercial or not-for-profit sectors.

\section{Declaration of interest}

None. 